\title{
Access to a Simulator is Not Enough: The Benefits of Virtual Reality Training Based on Peer-Group-Derived Benchmarks- A Randomized Controlled Trial
}

\author{
Martin W. von Websky • Dimitri A. Raptis • \\ Martina Vitz • Rachel Rosenthal • \\ P. A. Clavien - Dieter Hahnloser
}

Published online: 14 August 2013

(C) Société Internationale de Chirurgie 2013

\begin{abstract}
Background Virtual reality (VR) simulators are widely used to familiarize surgical novices with laparoscopy, but VR training methods differ in efficacy. In the present trial, self-controlled basic VR training (SC-training) was tested against training based on peer-group-derived benchmarks (PGD-training).

Methods First, novice laparoscopic residents were randomized into a SC group $(n=34)$, and a group using
\end{abstract}

Electronic supplementary material The online version of this article (doi:10.1007/s00268-013-2175-6) contains supplementary material, which is available to authorized users.

M. W. von Websky

Department of Surgery, University Hospital of Bonn, SigmundFreud Str. 25, 53127 Bonn, Germany

e-mail: martin@websky.de

D. A. Raptis · P. A. Clavien

Department of Visceral Surgery, University Hospital of Zurich, Raemistrasse 100, 8091 Zurich, Switzerland

M. Vitz

Laparoscopic Training Center, Seestrasse 323, 8038 Zurich,

Switzerland

R. Rosenthal

Department of Surgery, University Hospital of Basel,

Spitalstrasse 21, 4031 Basel, Switzerland

D. Hahnloser $(\square)$

Service de Chirurgie Viscérale, Centre Hospitalier Universitaire

Vaudois (CHUV), BH10/983 Rue du Bugnon 46,

1011 Lausanne, Switzerland

e-mail: dieter.hahnloser@chuv.ch
PGD-benchmarks $(n=34)$ for basic laparoscopic training. After completing basic training, both groups performed 60 VR laparoscopic cholecystectomies for performance analysis. Primary endpoints were simulator metrics; secondary endpoints were program adherence, trainee motivation, and training efficacy.

Results Altogether, 66 residents completed basic training, and 3,837 of 3,960 (96.8\%) cholecystectomies were available for analysis. Course adherence was good, with only two dropouts, both in the SC-group. The PGD-group spent more time and repetitions in basic training until the benchmarks were reached and subsequently showed better performance in the readout cholecystectomies: Median time (gallbladder extraction) showed significant differences of $520 \mathrm{~s}$ (IQR 354-738 s) in SC-training versus $390 \mathrm{~s}$ (IQR 278-536 s) in the PGD-group $(p<0.001)$ and $215 \mathrm{~s}$ (IQR 175-276 s) in experts, respectively. Path length of the right instrument also showed significant differences, again with the PGD-training group being more efficient.

Conclusions Basic VR laparoscopic training based on PGD benchmarks with external assessment is superior to SC training, resulting in higher trainee motivation and better performance in simulated laparoscopic cholecystectomies. We recommend such a basic course based on PGD benchmarks before advancing to more elaborate VR training.

$\begin{array}{ll}\text { Abbreviations } \\ \text { CC } & \text { Correlation coefficient } \\ \text { SD } & \text { Standard deviation } \\ \text { OR } & \text { Operating room } \\ \text { R } & \text { Range } \\ \text { IQR } & \text { Interquartile range } \\ \text { VR } & \text { Virtual reality }\end{array}$




\section{Introduction}

Virtual reality (VR) devices are widely accepted tools to familiarize surgical novices with the principles of laparoscopy without jeopardizing patient safety $[1,2]$. There is agreement that VR training is beneficial for many aspects of laparoscopic surgery, like hand-eye coordination, the fulcrum effect etc., and thus improves real operating room (OR) performance [3-5]. As a consequence, VR training is becoming increasingly popular, and many departments respond by purchasing a VR simulator. However, the best individually structured virtual training curricula are still a matter of intensive debate. Often, especially in basic training modules, only performance in partial tasks is trained (and studied), which quickly becomes tedious with diminishing trainee motivation. Moreover, it is hard to integrate VR training into the busy work week of a surgical resident [6]; therefore VR training must be efficient, and trainee frustration should be avoided. Otherwise, initial enthusiasm may be followed by discontinued use of VR simulators. One approach to tap the full potential of VR training is the concept of proficiency-based training as described by Ahlberg et al. [7], which is hypothesized to be superior to free training. The goal of proficiency-based training is to reach a predefined proficiency level instead of focusing on prescribed task repetitions or training sessions. This proficiency level is in most cases set by expert performance. In the cited study, trainees practiced under supervision, receiving expert and simulator feedback after each task [7]. However, in reality, expert performance on any given simulator can be hard to achieve for the novice, and qualified tutoring staff may not be available at all times. Concepts allowing for efficient, feasible, and well-accepted simulator training that is practiced by surgical residents with high intrinsic motivation are needed. Based on our experience with VR laparoscopic training, we hypothesized that simple access to a VR simulator and free, self-controlled (self-controlled) training of the surgical novice would not be sufficient-due to lack of stimulus and feedback - to efficiently achieve the training goals. Instead, the concept of feasible training based on peergroup-derived (PGD) benchmarks was hypothesized to be the better alternative to SC training, both because it is practical and at the same time avoids the possible frustration of not being able to reach expert performance.

Thus, the aims of the present study were to determine if a VR basic training course based on previously developed PGD benchmarks (subsequently referred to as "PGD" training/group B) combined with external formative assessment would be superior to free, SC training (subsequently referred to as "SC" training/group A) using the simulator metrics as primary- and trainee program adherence, trainee motivation and training efficacy as secondary-, endpoints.

\section{Materials and methods}

\section{Trainees}

During a 5-year period (2005-2010) surgical residents in Switzerland were randomized into two groups for basic laparoscopic training on the Simbionix LAP Mentor (see study flowchart, Fig. 1). Participating residents were recruited if their respective departments participated in a residential training program run by the Lapcenter Zurich and alternately randomized to the two study groups upon enrolment. Group A ( $n=34)$ trained freely (SC-training group) and group B $(n=34)$ followed a standardized curriculum based on peer-group benchmarks (PGD-training). Before the basic training modules started, both groups underwent identical familiarization with the simulator according to a standardized protocol (a $2 \mathrm{~h}$ demonstration and subsequent performance of each basic skill and procedural task one time under supervision). To stress the intent to train for safe surgery, both groups were instructed to avoid serious complications and unsafe cautery near to vital structures. Both groups then performed $60 \mathrm{VR}$ cholecystectomies over a time span of 1 year. For reference, their simulations were compared to results of experts $(n=3)$, who also performed 60 VR cholecystectomies on the simulator.

All trainees were novices in laparoscopic surgery, having performed fewer than five laparoscopic procedures. They were in their first or second year of training and had no previous VR simulator experience. Experts were experienced practicing laparoscopic surgeons with over 500 procedures in their log book.

Basic laparoscopic training and study metrics

The VR system used provides two sets of initial exercises that the trainees had to perform before attempting the VR cholecystectomies: (1) basic skills and (2) procedural tasks. The basic skills battery contained tasks for "camera manipulation with a $0^{\circ}$ scope," "camera manipulation with a $30^{\circ}$ scope," "eye-hand coordination," "clip applying," "clipping and grasping," "two-handed maneuvers," "cutting," "coagulation," and "object placement." The procedural task battery consists of "clipping and cutting," "clipping and cutting with two hands," "dissection," and "gallbladder separation," partly using the VR cholecystectomy environment (see Attachment 1 in Supplementary material). Primary endpoint metrics assessed by the simulator were as follows: time to complete the task, instrument pathways ( 1 and r), safe cautery, and serious complications as recorded by the software. Secondary endpoints related to training efficacy and trainee motivation were trainee adherence to the course, time spent on the simulator, and number of visits to the training facility. 
Fig. 1 Study flowchart

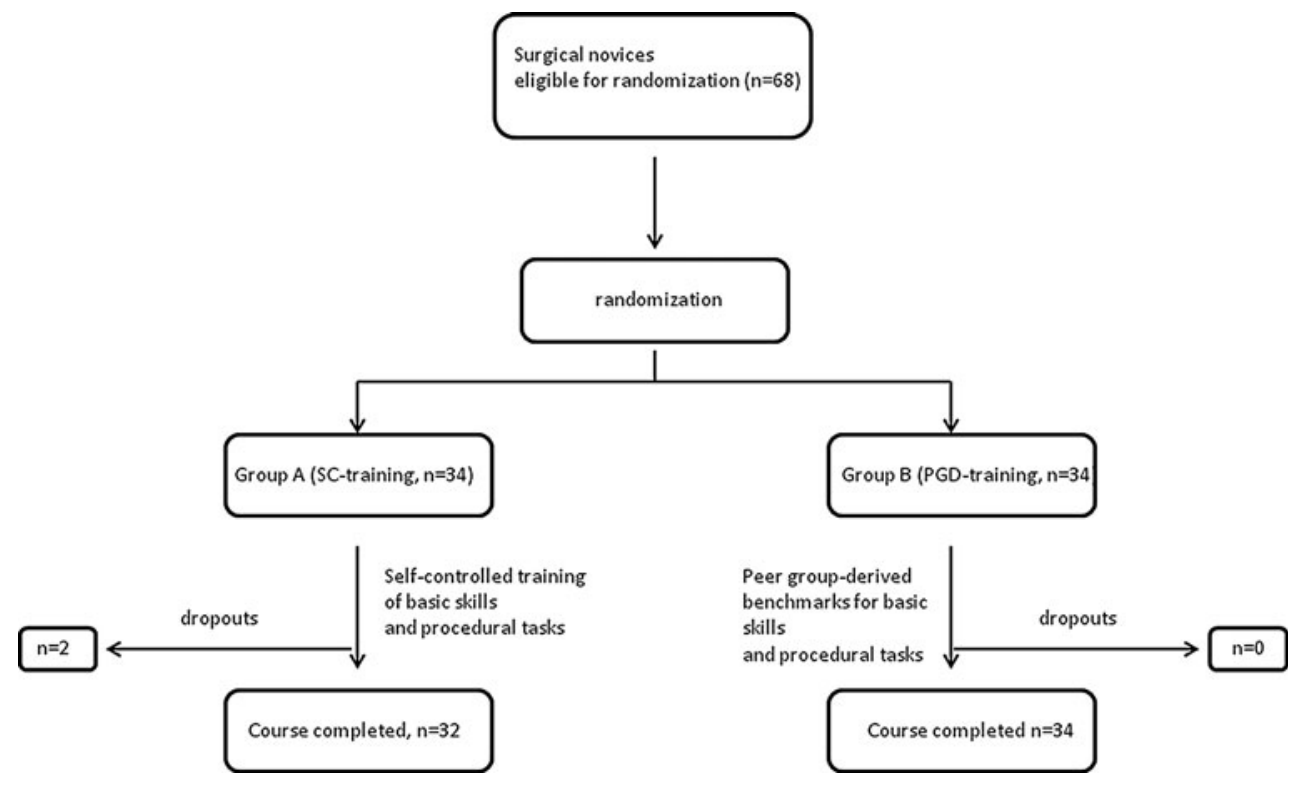

Study groups

The SC-training group (group A) started to practice the nine basic skills and the four procedural tasks until "confident" (via self-assessment). Upon request, an experienced instructor was available for assistance at all times. Once the trainees felt "confident," they were allowed to perform the full procedure cholecystectomies on the simulator.

The PGD-training group (group B) received a training curriculum with PGD benchmarks for the nine basic skills and the four procedural tasks until "proficient." "Proficiency" (i.e., the permission to stop basic training and begin with the VR cholecystectomies) in this case was defined as meeting these PGD benchmarks previously established on the basis of preliminary data from our group (after observing that the upper quartile of peer performance could serve as an effective training threshold, as outlined in our previous work [8]). "Proficiency" was then verified by the instructor (a process described as "external formative assessment"), and subsequently, the trainees were allowed to perform the VR cholecystectomies.

As readout, each group was encouraged to perform six different cases of VR cholecystectomy at least 10 times, each with somewhat variable anatomy, as preset by the simulator.

The study was funded by the Swiss National Foundation (SNF No: 32003B-120722) and registered at clinicaltrials.gov (Reg. No: NCT01615549). This investigator-initiated study was conducted without any financial support from Simbionix. Data were collected and analyzed in a prospective database. Performance parameters were recorded by the Simbionix LAP Mentor software, and output files were created in Microsoft Excel (Microsoft Corp., Redmond, WA).

\section{Statistics}

Statistical analysis was performed with PASW Statistics 20 (SPSS: An IBM Company, Chicago, IL; 2011). Continuous variables were non-normally distributed and compared with the Mann-Whitney $U$ test, and the Kruskal-Wallis $H$ test, as appropriate. The Dunnett T3 correction was used for multiple comparisons. All $p$ values were two-sided and considered statistically significant if $p<0.05$ [9-11]. To identify the learning curves, line fitting was performed for the measured parameters, and function types were determined based on the method of least squares with the use of eBiostatistics.com [12]. In all cases, the coefficient of determination $R^{2}$, which measures the quality of the fit, was calculated $\left(0<R^{2}<1\right)$. The type of curve that best combined plausibility and goodness of fit (i.e., coefficient of determination $R^{2}$ approaching one) was then chosen. Further information on the method of least squares may be found in Wolberg [13]. The Spearman's Correlation Coefficient test was used to identify which parameters showed the highest correlation.

\section{Results}

Trainee demographics

The median age of the trainees was 28 years (range $24-35$ years). $3 \%$ of the trainees were left hand dominant 
and $48 \%$ were women. There was no difference in laparoscopic or simulator experience between the two groups, as all were novices as defined above.

Data collection

Altogether, 66 residents completed the course, and 3,837 VR cholecystectomy cases were available for analysis, representing $96.8 \%$ of the predicted 3,960 procedures. Incomplete data sets resulting from technical failure were excluded, and some residents did not perform all of the 60 encouraged VR cholecystectomies. The SC-training group (group A) had two dropouts, and the remaining trainees $(n=32)$ performed 1,968 full cholecystectomy procedures. The PGD-training group B $(n=34)$ performed 1,869 procedures, and the expert group $(n=3)$ performed 145 procedures.

Primary endpoints: simulator metrics and performance in VR cholecystectomy

Time until extraction of the gallbladder was significantly different between the two novice groups A and B (see also Fig. 2). All groups were very good at observing safe distances from vital structures when using cautery. Here, no significant differences were found. Overall, most serious complications $(70 \%)$ occurred during the first three performances of simulated laparoscopic cholecystectomy (Fig. 3). The groups differed significantly regarding the occurrence of serious complications when compared to experts (experts: mean 0.1 serious complications per case [ $r$ 0-2, SD 0.31] versus group A: mean 0.51 serious complications per case $[r 0-21, \mathrm{SD} 1.21]$ versus group $\mathrm{B}$ : mean 0.44 serious complications per case $[r 0-14$, SD 0.97]; Kruskal-Wallis test: $p<0.001$ ), but not among themselves. The "spotlight analysis" at the tenth repetition of VR cholecystectomy verified that, at this point, the groups did not differ with regard to serious complications (Table 1).

Instrument pathways: correlation analysis

The pathways of the left and right instruments correlated highly with the time to extract the gallbladder (Spearman's $r$ between time to extract gallbladder and right instrument pathway, 0.89; Spearman's $r$ between time to extract gallbladder and left instrument pathway, 0.77). Also, the pathways of left and right instruments correlated highly among themselves (Spearman's $r$ between the left and right instrument pathways, 0.82). The instrument path length of the right instrument was significantly different in the training groups (group A: median right instrument pathway $633 \mathrm{~cm}$ [IQR 461-846 cm]; group B: median right instrument pathway $490 \mathrm{~cm}$ [IQR 364-665 cm]; Kruskal-

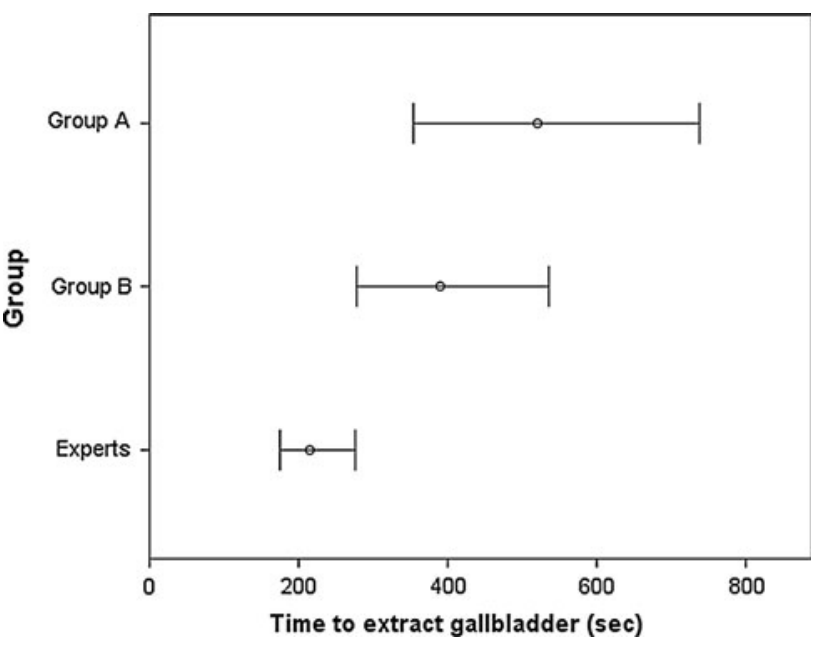

Fig. 2 Median time until gallbladder extraction by group (group A/SC-training: median $520 \mathrm{~s}$ (IQR 354-738 s) versus group B/PGDtraining: median $390 \mathrm{~s}$ (IQR 278-536 s); Kruskal-Wallis test: $p<0.001$, Mann-Whitney $U$ test: $p<0.001$. Experts: median $215 \mathrm{~s}$ (IQR 175-276). Horizontal lines represent the interquartile range (IQR); dot represents the median

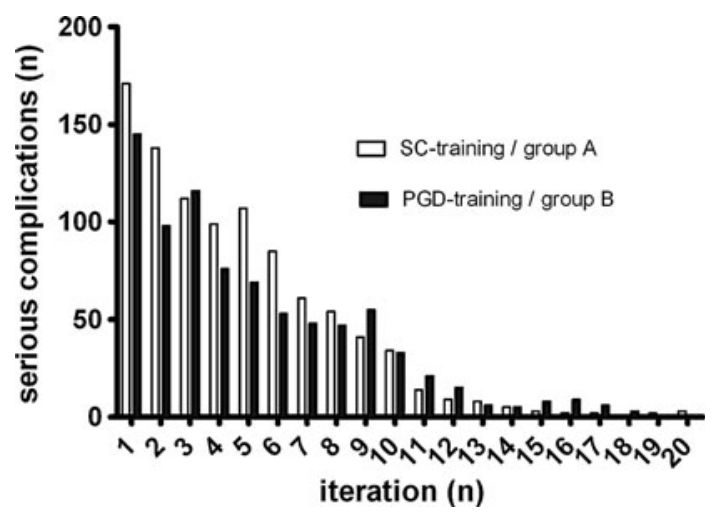

Fig. 3 Serious complications of group A (free training) and group B (proficiency-based training) according to iteration (1-20) of simulated cholecystectomy

Wallis test $p<0.001$, Dunnett's T3 $p<0.001$ ) and did not reach expert levels (experts: median right instrument pathway $375 \mathrm{~cm}$ (IQR 305-462 cm).

Learning curves

Learning curve characteristics were observed for the parameters "time to extract gallbladder" and "path length of the right instrument." The "safe cautery" parameter did not display learning curve characteristics. The differences in performance are visualized as learning curves (see also Fig. 4). Groups A and B reached the performance plateau for "time to extract the gallbladder" at the eighth and ninth repetitions, respectively. For "right instrument path length" the plateaus of group A and B were reached at the eleventh and seventh iterations, respectively. 
Table 1 Spotlight analysis at tenth repetition

\begin{tabular}{lll}
\hline Spotlight analysis at tenth repetition & $\begin{array}{l}\text { Group A-SC-training } \\
\text { (median) (IQR) } n=81\end{array}$ & $\begin{array}{l}\text { Group B-PGD-training } \\
\text { (median) (IQR) } n=107\end{array}$ \\
\hline Time to extract gallbladder $^{\mathrm{a}}$ & {$[429 \mathrm{~s}](356-575 \mathrm{~s})$} & {$[325 \mathrm{~s}](253-452 \mathrm{~s})$} \\
Serious complications $^{\mathrm{b}}$ & Mean: 0.4; SD: 0.7 & Mean: $0.3 ;$ SD: 0.7 \\
Safe cautery $^{\mathrm{b}}$ & {$[97 \%](92-100 \%)$} & {$[100 \%](92-100 \%)$} \\
Pathway right instrument $^{\mathrm{a}}$ & {$[567 \mathrm{~cm}](449-711 \mathrm{~cm})$} & {$[430 \mathrm{~cm}](349-539 \mathrm{~cm})$} \\
\hline
\end{tabular}

${ }^{a}$ Mann-Whitney $U$ test on $p<0.001$ level

b Nonsigificant

Per case analysis

A separate analysis of the six cholecystectomy cases available on the simulator revealed that in all cases (1-6) the PGD-training group (group B) performed significantly better than the SC-training group (group A) regarding time to extract the gallbladder and pathway of the right instrument. In cases 3 and 5, more serious complications were observed in total, most likely because of complications caused by an aberrant cystic artery in case 3 and an irregular accessory bile duct in case 5. The "per case" analysis data are available as supplementary material to provide benchmarks for the VR cholecystectomies (see Attachment 2 in Supplementary material).

Secondary endpoints: training efficacy and trainee motivation

The PGD-training group B invested more time, performed significantly more repetitions, and visited the training center more often to achieve the prescribed benchmarks in basic training than the SC-training group A (see Table 2). This resulted in markedly better performance in the readout procedure VR cholecystectomies, which were performed faster and more efficiently by group B. The overall total simulation time was equal between the two groups.

\section{Discussion}

In this randomized trial, we found that accessibility of a VR simulator, combined with free, self-controlled training ("SC-training") is not as effective and motivating as a training program using PGD benchmarks with external formative assessment ("PGD-training").

The advantages of simulated laparoscopic training comprise high face validity of the modern simulators, objective measurement of outcome parameters, and foremost optimal training opportunities with preservation of patient safety. Limitations of widespread use of VR are the following: First, the simulators are costly and not widely
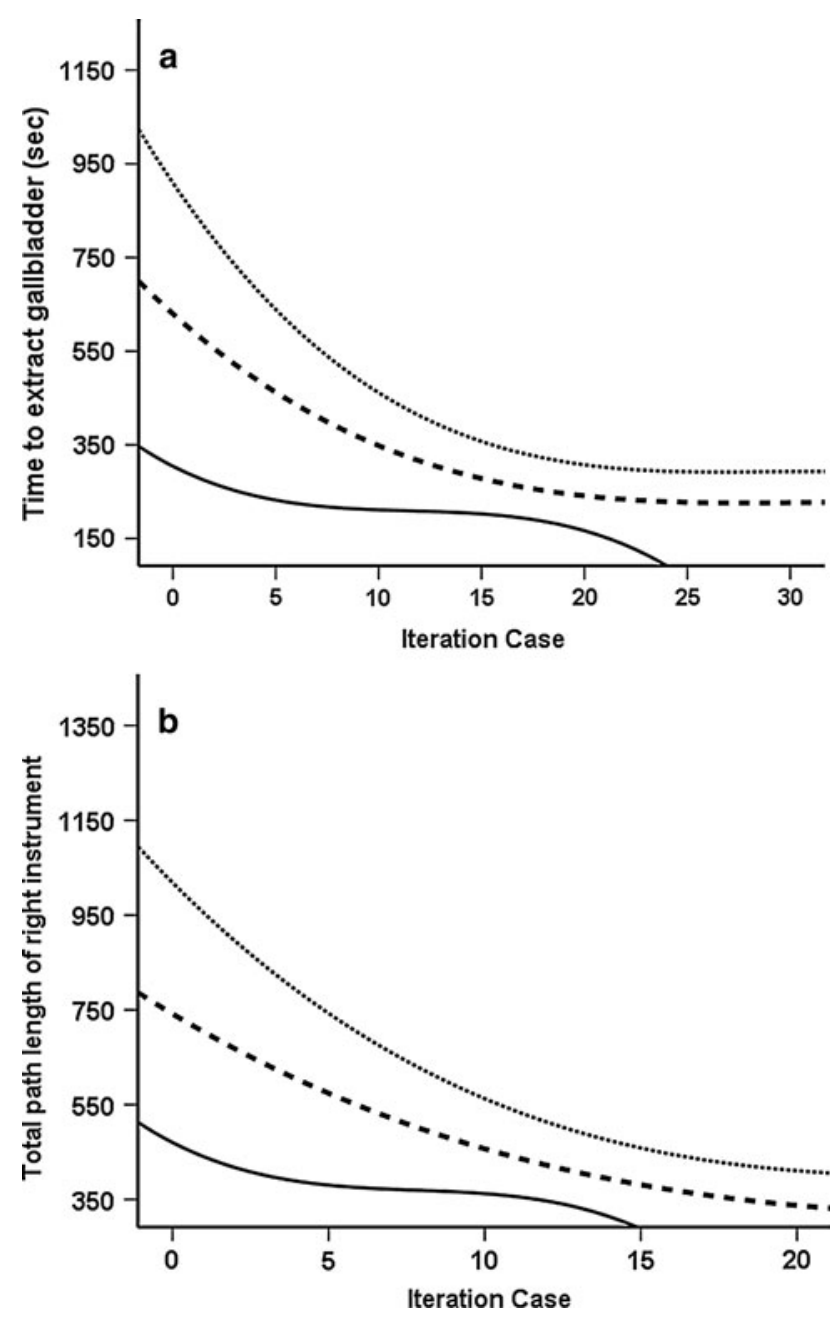

Fig. 4 a, b Learning curves for "time to extract gallbladder" and "path length right instrument." Upper interrupted lines represent group A (SC-training), middle interrupted lines represent group B (PGD-training), and lowest straight line represent experts. Multiple comparisons using Dunnett's T3 showed significant differences between all groups for "time to extract gallbladder" $(\mathbf{a} p<0.001)$ and "path length right instrument" (b $p<0.001)$

available enough to provide equal training opportunities for all young surgeons. Second, availability of a simulator in and of itself will not result in optimal training efficiency. 
Table 2 Practice times and visits in the training center

\begin{tabular}{lll}
\hline & Group A-SC-training (median) (IQR) & Group B-PGD-training (median) (IQR) \\
\hline Basic skills visits in training center $^{\mathrm{a}}$ & $4(2-6)$ & $6(4.8-8)$ \\
Performed repetitions $^{\mathrm{a}}$ & $61(37-85)$ & $104.5(74-165.8)$ \\
Total practice time $^{\mathrm{a}}$ & $2 \mathrm{~h} / 31 \mathrm{~min}(1 \mathrm{~h} / 49 \mathrm{~min}-4 \mathrm{~h} / 6 \mathrm{~min})$ & $4 \mathrm{~h} / 13 \mathrm{~min}(2 \mathrm{~h} / 48 \mathrm{~min}-6 \mathrm{~h} / 1 \mathrm{~min})$ \\
Procedural tasks visits in training center $^{\mathrm{a}}$ & $3(2-5)$ & $4.5(4-5.3)$ \\
Performed repetitions $^{\mathrm{a}}$ & $21(13-31)$ & $38(28-55.3)$ \\
Total practice time $^{\mathrm{a}}$ & $1 \mathrm{~h} / 27 \mathrm{~min}(1 \mathrm{~h} / 8 \mathrm{~min}-2 \mathrm{~h} / 10 \mathrm{~min})$ & $2 \mathrm{~h} / 6 \mathrm{~min}(1 \mathrm{~h} / 40 \mathrm{~min}-2 \mathrm{~h} / 32 \mathrm{~min})$ \\
Cholecystectomies visits in training center $^{\mathrm{a}}$ & $8(6-10.8)$ & $6(5-8)$ \\
Performed repetitions $^{\mathrm{a}}$ & $59(46.3-69.3)$ & $60.5(39.5-70.3)$ \\
Total practice time $^{\mathrm{a}}$ & $10 \mathrm{~h} / 12 \mathrm{~min}(8 \mathrm{~h} / 7 \mathrm{~min}-12 \mathrm{~h} / 57 \mathrm{~min})$ & $7 \mathrm{~h} / 5 \mathrm{~min}(5 \mathrm{~h} / 42 \mathrm{~min}-9 \mathrm{~h} / 15 \mathrm{~min})$ \\
Overall total practice time $^{\mathrm{b}}$ & $15 \mathrm{~h} / 30 \mathrm{~min}(12 \mathrm{~h} / 3 \mathrm{~min}-17 \mathrm{~h} / 56 \mathrm{~min})$ & $12 \mathrm{~h} / 39 \mathrm{~min}(11 \mathrm{~h} / 3 \mathrm{~min}-17 \mathrm{~h} / 54 \mathrm{~min})$ \\
\hline
\end{tabular}

${ }^{a}$ Mann-Whitney $U$ test, $p<0.001$

b Nonsignificant

Third, as "benchmark" data are not readily available for most simulators (unlike in commercial computer games where public "rankings" give the user an idea of his/her proficiency), formative and summative trainee assessment by instructors and design of basic training programs based on such data is difficult. As a consequence, performance in simulated laparoscopic training is not yet part of surgical curricula in many countries (a notable exception being the UK [14]). Thus, we aimed to prove that a basic training program based on PGD benchmarks with external assessment of proficiency (a) enhances performance in subsequent, more sophisticated tasks (VR cholecystectomy) and (b) ensures trainee adherence and motivation. To evaluate the effectiveness of the information presented here, we pose the following questions:

Which of the compared training methods was more effective in this study?

The two training groups in this study spent equal total time training on the simulator, and general acceptance of the simulator platform was high, as only two dropouts were recorded (both in SC-group A). The PGD-group B, practiced more to complete the somewhat "tedious" basic skills and procedural tasks before they reached the preset benchmarks and were allowed to proceed to the simulated cholecystectomies. The trainees in the SC-group A quickly felt (falsely) confident and moved on to the readout procedures. Group A then had to spend more time when attempting the VR cholecystectomies, because they were slower and less experienced. In addition, they showed a persisting inferior learning curve to that of the PGD-group $\mathrm{B}$ in several simulator metrics (Fig. 4), demonstrating that the SC basic training approach was less effective. Most likely, the necessity to "gain the reward" of being allowed to perform the VR cholecystectomies by passing the required benchmarks resulted in a higher motivation and enhanced basic training efforts in group B $[15,16]$. This finding is in line with a previous study by Halvorsen et al. [17] who showed that unsupervised VR training did not improve laparoscopic suturing skills.

What is the difference between "PGD benchmarks" and "proficiency-based training"?

Normally, proficiency-based training describes the use of benchmarks set by expert performance for the training of surgical novices [18]. This has the potential disadvantage of creating a large gap between expected performance and individual training experience of novices and can lead to trainee frustration, as expert competency might not be achievable in the allotted training time frame. Van Dongen et al. [19] used thresholds based on mean expert scores plus twice the standard deviation to establish feasible criteria for trainees in a consensus paper on the LapSim simulator. In a previous work, we showed the efficacy of using PGD benchmarks for basic laparoscopic training on the Simbionix Lap Mentor by using the best quartile of performance in the peer group as the proficiency benchmark in the respective task [8]. Consequently, it had to be determined whether these PGD benchmarks result in better performance in an advanced laparoscopic task. It is evident from our data that neither novice group could reach expert performance in VR cholecystectomy on the simulator, but the PGD-group B trained more efficiently and performed better than the SC-group A in most measured parameters.

Which parameters and benchmarks should be used for trainee assessment?

Because the intention to train for safe surgery is paramount, instructors should emphasize the importance of 
observing safe laparoscopic practices. In our experience, this prioritization resulted in a high level of "safe cautery" and fast reduction of "serious complications" over time in both groups. The "safe surgery" principle is a conditio sine qua non for all laparoscopic training and can be used as a quality parameter of trainee performance. Measures of proficiency and trainee progress can comprise time to task completion and instrument pathways (always with the caveat that safety parameters stay in range or improve), but not every trainee will be able to reach a satisfactory competency level [20]. As our correlation analysis shows, especially the right instrument pathway (working hand) predicts time to task completion and displays the operator's efficiency of movement. To provide benchmark data for reference, our descriptive parameter data for VR cholecystectomy cases 1-6 on the Simbionix Lap Mentor are available on request (see Attachment 1 in Supplementary material).

What principles should be observed when installing a VR laparoscopic training program?

The key message of the present study is that it is advisable to design a basic training course based on task-specific proficiency criteria and install a dedicated instructor team. The proficiency criteria may vary with the simulator and tasks, and even with the trainee collective. In our view "peer group-derived benchmarking" (using the 75th percentile of peer performance in the respective task as proficiency benchmark) may be practical in designing motivating and feasible VR training. Furthermore, it is necessary to provide sufficient stimulus to participate in the courses. For example, it could be considered to allow only novices that have successfully participated in a basic laparoscopic training course and passed external assessment by an instructor to be assigned to real-life laparoscopy in the OR.

What are the limitations and strengths of this study?

Our study provides evidence that benchmark-based basic training coupled with external assessment results in efficient preparation for more sophisticated laparoscopic simulation. As with every study, there are some limitations to consider when interpreting our data: First, the VR device used for basic training and readout VR cholecystectomy was identical, so that transferability of skills among VR systems was not assessed, as has already been done in other studies [21]. Second, transferability of results to real OR performance was not addressed in this study - this is still a challenge for ongoing research. On a different simulator, the LapSim, Gauger et al. [22] demonstrated improved novice performance using task-specific proficiency criteria much the same way as in our approach; however, a transferability demonstration to real OR performance failed because of the sample size. Third, our study showed that efficiency parameters benefited more from our intervention than the above-mentioned "safety parameters." We are not sure why this is the case. Arguably this reflects our instruction and course philosophy (as both groups were equally good at observing the safety parameters), and thus the effect of PGD training on the safety parameters was not detectable in this setting. This is an aspect that may deserve further study.

\section{Conclusions}

In the present study we have shown that SC training (with self-assessment) on a VR simulator is not as efficient as basic training based on PGD benchmarks with external formative assessment. With equal time budgeted, the PGDtraining method results in better performance in VR cholecystectomy. Appropriate assessment parameters along with benchmark data are provided for design of such basic laparoscopic training courses, which can form a cornerstone of future resident training. Ongoing studies are needed to demonstrate transferability of virtually acquired laparoscopic skill to the OR.

Acknowledgments This study was funded by the Swiss National Foundation (SNF No: 32003B-120722) and is registered at http:// www.clinicaltrials.gov (Reg. No: NCT01615549). The authors are grateful to Andreas Scheiwiller, Ralph Stärkle, and Jêrome Gapany for their professional support, as well as to the Frieda-Locher-Hofmann Foundation for continuing engagement in establishing laparoscopic simulator training for young surgeons.

\section{References}

1. Ganai S, Donroe JA, St Louis MR et al (2007) Virtual-reality training improves angled telescope skills in novice laparoscopists. Am J Surg 193:260-265

2. Kohn L, Corrigan J, Donaldson M (1999) To err is human: building a safer health system. A report of the National Institute of Medicine, Washington, DC, The National Academies

3. Grantcharov TP, Kristiansen VB, Bendix J et al (2004) Randomized clinical trial of virtual reality simulation for laparoscopic skills training. Br J Surg 91:146-150

4. Seymour NE, Gallagher AG, Roman SA et al (2002) Virtual reality training improves operating room performance: results of a randomized, double-blinded study. Ann Surg 236:458-463 discussion 463-464

5. Andreatta PB, Woodrum DT, Birkmeyer JD et al (2006) Laparoscopic skills are improved with Lap Mentor training: results of a randomized, double-blinded study. Ann Surg 243:854-860 discussion 860-863

6. Stefanidis D, Acker CE, Swiderski D et al (2008) Challenges during the implementation of a laparoscopic skills curriculum in a busy general surgery residency program. J Surg Educ 65:4-7 
7. Ahlberg G, Enochsson L, Gallagher AG et al (2007) Proficiencybased virtual reality training significantly reduces the error rate for residents during their first 10 laparoscopic cholecystectomies. Am J Surg 193:797-804

8. von Websky MW, Vitz M, Raptis DA et al (2012) Basic laparoscopic training using the Simbionix LAP Mentor: setting the standards in the novice group. J Surg Educ 69:459-467

9. Siegel S, Castellan NJ (1988) Nonparametric statistics for the behavioral sciences. McGraw-Hill, New York

10. Conover WJ (1980) Practical nonparametric statistics, 2nd edn. Wiley, New York

11. Daniel WW (1995) Biostatistics, 6th edn. John Wiley and Sons, New York

12. eBiostatistics.com: function approximation. http://www.eBiostatistics. com. Accessed 3 Aug 2013

13. Wolberg J (2005) Data analysis using the method of least squares: extracting the most information from experiments. Springer, New York

14. The National Training Programme in Laparoscopic Colorectal Surgery. http://www.lapco.nhs.uk/about-the-programme.php. Accessed 3 Aug 2013

15. Ricci K, Salas F, Cannon-Bowers J (1996) Do computer based games facilitate knowledge acquisition and retention? Mil Psychol 8:295-307
16. Garris R, Ahlers R, Driskell J (2002) Games, motivation and learning: a research and practice model. Simul Gaming 33: 441-467

17. Halvorsen FH, Fosse E, Mjaland O (2011) Unsupervised virtual reality training may not increase laparoscopic suturing skills. Surg Laparosc Endosc Percutan Tech 21:458-461

18. Heinrichs WL, Lukoff B, Youngblood P et al (2007) Criterionbased training with surgical simulators: proficiency of experienced surgeons. JSLS 11:273-302

19. van Dongen KW, Ahlberg G, Bonavina L et al (2011) European consensus on a competency-based virtual reality training program for basic endoscopic surgical psychomotor skills. Surg Endosc $25: 166-171$

20. Grantcharov TP, Funch-Jensen P (2009) Can everyone achieve proficiency with the laparoscopic technique? Learning curve patterns in technical skills acquisition. Am J Surg 197:447-449

21. Hamilton EC, Scott DJ, Fleming JB et al (2002) Comparison of video trainer and virtual reality training systems on acquisition of laparoscopic skills. Surg Endosc 16:406-411

22. Gauger PG, Hauge LS, Andreatta PB et al (2010) Laparoscopic simulation training with proficiency targets improves practice and performance of novice surgeons. Am J Surg 199:72-80 